\title{
Correction to: Orientation constraints for Wi-Fi SLAM using signal strength gradients
}

\author{
Hsiao-Chieh Yen $^{1}$ (D) $\cdot$ Chieh-Chih Wang ${ }^{2} \cdot$ Cheng-Fu Chou $^{1}$
}

Published online: 27 August 2020

๑) Springer Science+Business Media, LLC, part of Springer Nature 2020

\section{Correction to:}

\section{Autonomous Robots}

https://doi.org/10.1007/s10514-020-09914-z

The original version of this article unfortunately missing the "Acknowledgements" section.

The "Acknowledgements" is given below:

Acknowledgements This work was supported by grants from Ministry of Science and Technology of Taiwan (\#109-2634-F-002 -024 -, \#1092221-E-002 -149 -MY3, \#109-2634-F-002 -030 -, \#109-2218-E-002 -017 -).

Publisher's Note Springer Nature remains neutral with regard to jurisdictional claims in published maps and institutional affiliations.

Hsiao-Chieh Yen

d97922035@ntu.edu.tw

Chieh-Chih Wang

bobwang@nctu.edu.tw

Cheng-Fu Chou

ccf@csie.ntu.edu.tw

1 Department of Computer Science and Information Engineering, National Taiwan University, Taipei, Taiwan

2 Department of Electrical and Computer Engineering, National Chiao Tung University, Hsinchu, Taiwan 